\title{
Immunotherapy in ALL: from Bench to
}

\section{Bedside}

\section{Dr. Rania Abdel-Monem Radwan, MD}

\section{Lecturer of Internal Medicine, Department of Hematology and Bone Marrow transplantation, Ain Shams University}

\section{Introduction:}

Overall survival for acute lymphoblastic leukemia (ALL) in children exceeds $85 \%$. Improved survival primarily stems from decreased incidence of relapse, with very little improvement for more than 20 years in survival rates for children who relapse. In contrast, overall survival for adults with ALL is quite poor (30\% to $40 \%) .{ }^{1}$ Recent data indicate that the use of more intensive pediatric regimens in adults can improve outcomes somewhat, ${ }^{2}$ but the high-risk features associated with B-ALL in adults makes it unlikely that the successes seen in children will be achieved with currently available regimens. Treatment of relapsed B-ALL also remains a substantial challenge, with outcomes varying depending on several factors, including age and length of first remission. Allogeneic hematopoietic stem cell transplantation (allo-HSCT) leads to cure in $\sim 50 \%$ of patients who achieve second complete remission. ${ }^{3}$ However, substantial numbers of patients do not successfully reach a second complete remission and therefore are not candidates for this therapy. As a result, data in which all relapses are included in the analysis irrespective of whether allo-HSCT is performed show a much more dismal overall survival for patients with relapsed $A L L$, despite intensive, highly toxic therapy. Furthermore, all salvage therapies currently available for B-ALL are associated with substantial short-term and long-term toxicity. Therefore, Novel treatment modalities are needed. ${ }^{4}$

The modern era of genomics has identified several potential molecular targets in BALL and the addition of bcr-abl inhibitors to standard cytotoxic regimens in Philadelphia chromosome-positive ALL markedly improve the outcome in this high-risk subgroup. ${ }^{5}$ However, more limited success has been achieved using inhibitors of other candidate targets such as JAK2, ${ }^{6}$ likely due to the multitude of pathways that contribute to oncogenesis in this disease. ${ }^{7}$ The emerging picture of B-ALL oncogenesis suggests that effective targeting of BALL cell surface molecules using mAb-derived therapeutics will be challenging. ${ }^{8}$ 


\section{Immunotherapy rationale:}

Potentially malignant cells are continuously eliminated by apoptosis and the immune system, but cancers have escaped these mechanisms. For immune-mediated clearance of leukemia to be possible, tolerance has to be overcome. This is the basis for the graft-versusleukemia (GVL) effect, which contributes in part to the efficacy of allogeneic stem cell transplantation (SCT) and is the rationale for donor lymphocyte infusion in leukemia. ${ }^{9}$

Recently, a new approach that uses genetic engineering to endow $T$ cells with receptors that bind leukemia cell surface antigens has shown increasing promise.

\section{Optimizing CAR-based therapeutics for B-ALL}

Chimeric antigen receptors (CARs) are synthetic receptors comprised of three key components (Fig. 1): (1) an extracellular antigen-binding domain derived from a monoclonal antibody single chain variable fragment (scFv); (2) a transmembrane linking domain derived from CD3, CD4, CD8 or CD28; (3) an intracellular signal domain consists of $C D 3 \zeta$ with or without costimulatory molecules. DNA constructs encoding such CARs could be stablely incorporated into human $T$ cells via lentiviral or gamma-retroviral transductions, electroporation, as well as transposon. ${ }^{10}$

The native TCR-CD3 complex is composed of 6 separate chains $(\alpha, \beta, \gamma, \delta, \epsilon$, and $\zeta)$, but signals generated via the $\zeta$ chain alone are sufficient to induce downstream events that are indistinguishable from those generated by an intact TCR. ${ }^{11}$ Most first generation CARs utilized $\mathrm{CD} 3 \zeta$ as the only stimulatory molecule to activate $T$ cells, which ultimately revealed defects of weak proliferation ability, poor anti-tumor effect, and short survival of T cells. ${ }^{12}$

The second and third generation CARs introduced one or two costimulatory signal domains, (Fig. 2) which significantly enhanced the expansion, persistence and potency of CAR-T cells. Costimulatory molecules can be CD28, 4-1BB, CD22, CD27, OX40, or ICOS, among which CD28 and 4-1BB are currently the most widely used. Studies showed that CD28 endued CAR-T cells with stronger killing ability, while 4-1BB granted longer persistence in vivo Advances of CD19directed chimeric antigen receptor-modified $T$ cells in refractory/relapsed acute lymphoblastic leukemia. ${ }^{13}$

\section{Effector cell screening}

The ideal effector cell type for CAR technology should have the following characteristics: (1) amplification can be achieved to meet the requirements of adoptive infusion both in vivo and in vitro; (2) sufficient tumor killing ability; (3) ability to mobilize to the tumor site; (4) predictable and manageable side effects. ${ }^{14}$ As the major component of 
cellular immunity, T lymphocytes satisfy all the above characteristics, and thus are most widely used as effector cells for CAR technology. ${ }^{15}$

CAR-T cells specifically recognize tumor cell surface antigen through antigen-antibody reaction and are independent of major histocompatibility complex (MHC), therefore avoiding tumor's immune escape by down-regulation of MHC. Turtle et al. found that CAR-T cells manufactured from central memory $T$ cells or initial T cells had stronger tumor-killing ability than those from effector memory cells. ${ }^{16}$ CAR-T cells from different T lymphocyte subsets will be a hotspot in future research.

\section{Antigen selection}

Ideally, an antigen targeted by CAR-modified T cells would be tumor specific. Beyond that, an ideal antigen would be ubiquitously expressed on tumor cells, but not expressed on normal cells. This requires a cell-surface molecule that is unique to tumor cells, perhaps through altered expression, translocation producing a fusion protein, or mutation leading to altered configuration or antibody binding. Such unique antigens are hard to find. But viable alternatives include antigens that are expressed on a single cell lineage whose function is dispensable or replaceable or antigens that are differentially expressed on tumor cells compared with normal cells. ${ }^{17}$

CD19 is a B-cell surface protein expressed throughout B-cell development; therefore, it is expressed on nearly all B-cell malignancies, including chronic lymphocytic leukemia (CLL), ALL, and many non-Hodgkin lymphomas. ${ }^{18}$ This near-universal expression and specificity for a single cell lineage has made CD19 an attractive target for CAR-modified T-cell therapies. Additional B-cell-specific cell-surface molecules, such as CD22, may hold similar promise and are under active investigation. ${ }^{19}$

Another potentially important feature of CAR target selection is consideration of the functional significance of the surface protein for survival of the malignant cell. CD19 and CD22 are important components of the BCR complex and there are emerging data showing that CD19 contributes to oncogenesis as a downstream mediator of PAX5, which augments MYC expression. $^{20}$

Target identification for T-cell ALL poses a particular challenge as leukemic blasts express the same antigens as normal T cells; therefore, CAR immunotherapy may not be possible in T-cell ALL. This is because B-cell aplasia is treatable and tolerable, whereas T-cell aplasia is not. Although select T-cell ALL subsets may aberrantly express cell-surface proteins that are not normally expressed on T cells or express abnormal fusion proteins, there is no universal target that is specific to T lymphoblasts. ${ }^{17}$ 


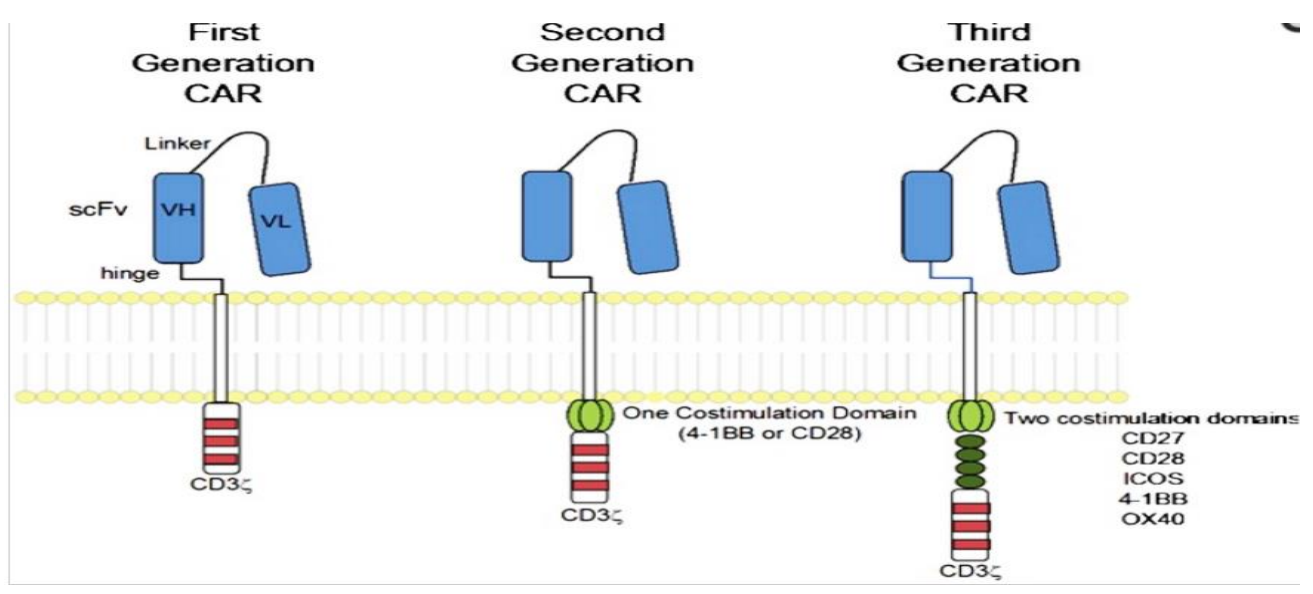

Fig. (1): CAR structure, according to signaling domains. CAR molecules link an extracellular single-chain variable fragment (scFv) to intracellular signaling domains. The intracellular component includes the CD3 3 intracellular signaling domain of the T-cell receptor either alone (first generation) or in combination with 1 (second generation) or 2 (third generation) costimulatory domains. ${ }^{10}$

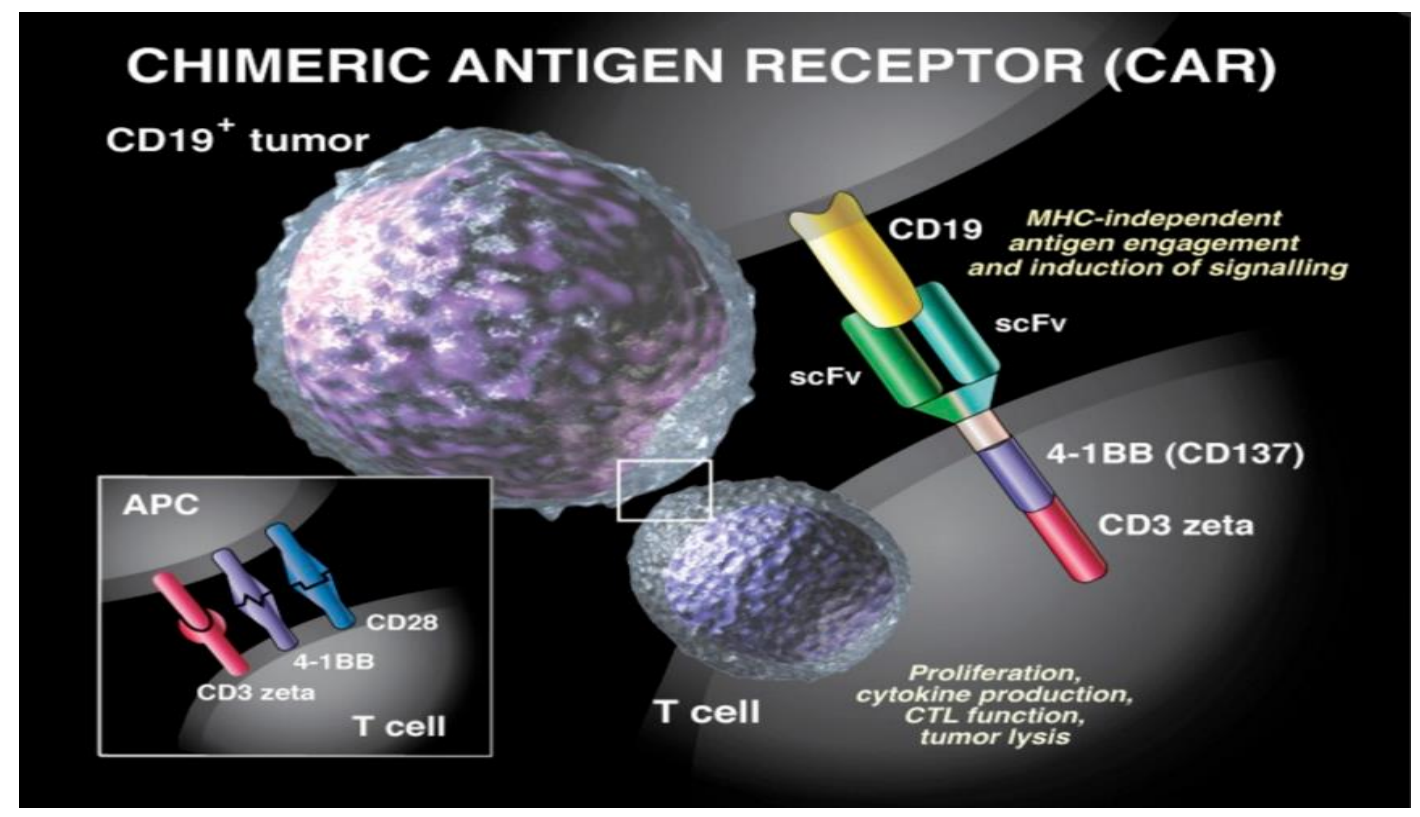

Fig. (2): Second-generation CAR used in current clinical studies at Penn and CHOP. CTL, cytotoxic T lymphocyte; MHC, major histocompatibility complex. ${ }^{13}$

\section{Clinical application of CD19 CAR-T therapy}

One important aspect of CAR therapy is that, unlike antibody-based regimens, which mediate antitumor effects for only as long as the antibody remains present in the host, CD19CAR T cells undergo dramatic expansion after infusion in response to CD19 antigen expressed on malignant and nonmalignant cells. Genetically modified CAR-expressing T cells can also persist for several months or even years. ${ }^{21}$ Therefore, CAR T cells represent a dynamic therapy, which is well illustrated by the fact that both response and toxicity are often delayed for several days after cell infusion. ${ }^{22}$ Finally, CAR T cells traffic to multiple tissue sites, including the $\mathrm{CSF}^{23}$ an important consideration in $\mathrm{B}-\mathrm{ALL}$, in which CNS relapse is a substantial risk. 
Clinical application of CD19 CAR-T cells was first pursued in the phase I clinical trial of MSKCC, in succession followed by $\mathrm{NCl}$ and UPENN. The CR rate for refractory/relapsed ALL were $88 \%, 67 \%$ and $90 \%$, respectively, much higher than traditional chemotherapy. Since then, numerous studies have been carried out across the globe with similar encouraging results. ${ }^{24}$ Despite the promising results, the conditioning regimen and the infusion dose differ among these studies. Joint efforts are urgently needed to form a standard protocol. ${ }^{13}$

\section{Toxicity and challenges}

\section{- Cytokine release syndrome (CRS)}

The most common and potentially severe toxicity associated with CAR-modified T-cell therapy is CRS. ${ }^{25}$

Cytokine release syndrome (CRS) is an inflammatory process related to exponential Tcell proliferation with resultant marked elevations in cytokine levels. Symptoms can range from mild flu-like symptoms to shock and multisystem organ failure. The cytokine profile after CAR-modified T-cell therapy mirrors the same profile seen in macrophage activation syndrome/ hematophagocytic-lymphohistiocytosis (MAS/HLH). ${ }^{26}$

CRS includes marked elevations in soluble interleukin-2 receptor $\alpha$ (sIL2Ra), interleukin-6 (IL-6), IL-10, and interferon (IFN- $\gamma$ ). Moreover, patients who develop severe CRS after CAR-modified T cells often develop clinical and laboratory manifestations similar to MAS/HLH, including marked hyperferritinemia (>10000 ng/mL), hepatomegaly/splenomegaly, and hypofibrinogenemia (<150 mg/dL). ${ }^{27}$ We hypothesize the IFN- $\gamma$ produced by the CAR-modified T cells may be driving the secondary MAS/HLH in patients with severe CRS. ${ }^{28}$

Some reports suggest amelioration of symptomatology after treatment with corticosteroids. One case report suggested that neutralizing antibody to soluble IL-6R may mitigate this inflammatory syndrome, ${ }^{22}$ although it is too early to know whether such treatment also limits the efficacy of the therapy.

Early data suggest that there may be a correlation between the development of CRS and the response to therapy; patients who do not develop CRS may be less likely to benefit from CAR-modified T cells, whereas those who develop CRS often respond to the therapy. Although there may be some correlation between developing CRS and efficacy, there does not appear to be a strong correlation between the degree of CRS and response to therapy. This is because of the confounding and strong impact of disease burden on the risk of severe CRS. $^{27}$ 


\section{- Encephalopathy}

Neurologic toxicities have been reported after T-cell-engaging therapies, including distinct CAR-modified T-cell therapies, Global encephalopathy is the most common toxicity, but seizures have also been reported. ${ }^{29}$ Scans (computed tomography and/or magnetic resonance imagine) and lumbar punctures have revealed no etiology of this syndrome, although CAR T cells are seen in the spinal fluid of most patients, regardless of encephalopathy. As we have seen encephalopathy after administration of tocilizumab, it does not appear to be prevented by IL- 6 blockade. Larger studies are needed to further characterize the spectrum of neurotoxicity with CAR therapies as well as the pathophysiology. ${ }^{17}$

\section{- B-cell aplasia}

Chronic B-cell aplasia, and resultant hypogammaglobulinemia, is an expected ontarget toxicity of successful CD19-directed CAR T-cell therapy. CD19 CAR therapies eliminate normal mature and precursor B cells. As long as CAR-modified T cells persist, B-cell aplasia continues, which provides what appears to be a highly accurate pharmacodynamic marker of CAR function. Although immunoglobulin replacement mitigates most infectious complications, longer follow-up is needed to assess late toxicity of B-cell aplasia. ${ }^{17}$

Strategies to eradicate CAR-expressing T cells using suicide vectors are being tested as one approach to preventing such long-term toxicity, but no results are available thus far using this approach. Suicide vectors have also been proposed as a means to prevent acute toxicity such as cytokine release syndrome, although it remains unclear whether one can retain potent antitumor effects if the cells are induced to undergo apoptosis early after administration. ${ }^{8}$

It remains possible that leukemia eradication could be accomplished without prolonged persistence of CAR T cells, in which case B-cell recovery is likely. Indeed, early results from some clinical groups suggest that potent antitumor effects can occur with CD19-based therapies followed by B-cell recovery. ${ }^{8}$

\section{Challenges for CD19 CARs}

CD19 CAR T cells have shown remarkable promise in ALL. Notwithstanding that success, challenges remain. For CAR T-cell therapies to be available to more patients, the delivery of this therapy needs to be feasible across institutions. Expanding access will require comprehensive training of clinicians and a standardized approach to CRS grading and management. ${ }^{30}$ 
Additional studies aimed at minimizing serious toxicities will be important as this therapy is exported to more sites. Across studies, disease burden appears to be correlated with CRS severity in responding patients. Although disease burden is difficult to modify in a highly refractory population, prophylactic measures, such as early tocilizumab administration, may prove effective in mitigating serious toxicities. ${ }^{17}$

An additional potential issue that has been suggested by some of the experiences thus far is that the collection, activation, and transduction of T cells may be more challenging in patients with B-ALL due to the potently immunosuppressive properties of ALL regimens currently in use. Indeed, transduction efficiencies appear to be lower in T cells collected from B-ALL patients compared with those collected from chronic lymphocytic leukemia patients using the same expansion process and vectors. One could potentially address this issue by harvesting $T$ cells earlier in the disease process and cryopreserving them for potential use in CAR-based adoptive T-cell therapy at a later time if clinically appropriate. Another option is to optimize ex vivo expansion protocols for heavily pretreated patients. ${ }^{8}$

Finally, relapse after CAR T-cell therapy remains a challenge. Two modes of disease recurrence have been seen: CD19 positive and CD19 negative. Relapse of ALL that retains surface CD19 expression results from rapid disappearance of CAR-modified T cells or decreased function of those T cells. Optimized CAR designs, manufacturing technologies, or T-cell subset ratios may prevent some of these relapses by prolonging T-cell persistence. ${ }^{17}$

Single-target therapy may select for and lead to escape variants. Combination or tandem CARs, which join 2 antigen-recognition moieties, may prevent relapses due to escape variants but need further studies. In this regard, the group at the National Institutes of Health has developed CAR T cells targeting the B-cell antigen CD22, ${ }^{19}$ which can be used for treating CD19-negative relapse and could be combined with a CD19-directed CAR in the future.

\section{Conclusions}

CD19-targeted CARs have paved the way for engineered T-cell therapies with high response rates and durable remissions reported. These results are unprecedented in patients without curative options. As new innovations in CAR design and manufacture develop and toxicity management evolves, the potential uses for this therapy will be expanded, as will access. 


\section{Expectation}

The 58th ASH annual meeting illustrated the eminent work that researchers had been doing in CAR-T cell therapy. Shah' team developed new CAR-T cells targeting CD22, which showed encouraging results in B-cell precursor acute lymphoblastic leukemia, ${ }^{31}$ suggesting that CD22 CAR-T cells may be a joint or remedial therapy for CD19-negative ALL and CD19-negative relapses. CD38 and CD123 may also be two important targets for refractory/relapsed ALL, as the former could reduce ontarget off-tumor effects and the latter shows a strong anti-tumor effect on CD19-negative ALL mice in preclinical trials. ${ }^{32}$

Avanzi et al. demonstrated that CD19 CAR-T cells that constitutively secrete IL-18 significantly increased serum cytokine secretion, enhanced CAR-T cell persistence, induced long-term B cell aplasia and improved mouse survival, even without any prior preconditioning. ${ }^{33}$ In addition, multiple centers are trying to develop new types of CAR-T cells, including universal CAR-T cells and hematopoietic stem cell derived CAR-T cells. De Oliveira et al. has confirmed the feasibility of incorporating CARs into hematopoietic stem cells. These modified stem cells can persist and differentiate into multiple branches of specific immune cells harboring CAR, thus effectively reducing relapses. ${ }^{34}$

Moreover, combination therapies using CAR-T cells and other medications such as bortezomib, lenalidomide, tyrosine kinase inhibitors, immune checkpoint antibodies, and cytokines may show interesting results. One study found that lenalidomide has synergistic anti-tumor effects with CD19 CAR-T cells and CD20 CAR-T cells in treating B-Non Hodgkin's lymphoma (B-NHL). ${ }^{35}$

We expect the safety and effectiveness of CD19 CAR-T cells to be further improved in the future. Through optimal designs of CAR-T cells, establishing standard protocol for conditioning and infusion, better control of complications, and combination with other therapeutic options, CD19 CART cells will provide a new or the ultimate solution for the treatment of refractory/relapsed ALL.

\section{References}

1- Bhojwani D, Pui C-H. Relapsed childhood acute lymphoblastic leukaemia. Lancet Oncol. 2013;14(6):e205e217.

2- Huguet F, Leguay T, Raffoux E, et al. Pediatric-inspired therapy in adults with Philadelphia chromosomenegative acute lymphoblastic leukemia: the GRAALL-2003 study. J ClinOncol. 2009; 27(6):911-918.

3- $\quad$ Leung W, Campana D, Yang J, et al. High success rate of hematopoietic cell transplantation regardless of donor source in children with very high-risk leukemia. Blood. 2011;118(2):223-230.

4- Forman SJ, Rowe JM. The myth of the second remission of acute leukemia in the adult. Blood. 2013; 121(7):1077-1082.

5- Schultz KR, Bowman WP, Aledo A, et al. Improved early event-free survival with imatinib in Philadelphia chromosomepositive acute lymphoblastic leukemia: a children's oncology group study. J Clin Oncol. 2009;27(31):5175-5181. 
6- Weigert O, Lane AA, Bird L, et al. Genetic resistance to JAK2 enzymatic inhibitors is overcome by HSP90 inhibition. J ExpMed. 2012;209(2):259-273.

7- Roberts KG, Morin RD, Zhang J, et al. Genetic alterations activating kinase and cytokine receptor signaling in high-risk acute lymphoblastic leukemia. Cancer Cell. 2012;22(2):153-166.

8- Fry TJ, Mackall CL. T-cell adoptive immunotherapy for acute lymphoblastic leukemia. ASH Education Book December 6, 2013; 2013(1): 348-353.

9- Loren AW, Porter DL. Donor leukocyte infusions for the treatment of relapsed acute leukemia after allogeneic stem cell transplantation. Bone Marrow Transplant. 2007;41(5):483-493.

10- Davila ML, Sadelain M. Biology and clinical application of CAR T cells for B cell malignancies. Int J Hematol. 2016; 104(1):6-17.

11- Irving BA, Weiss A. The cytoplasmic domain of the T cell receptor zeta chain is sufficient to couple to receptor-associated signal transduction pathways. Cell. 1991;64(5):891-901.

12- Carpenito C, Milone MC, Hassan R, et al. Control of large, established tumor xenografts with genetically retargeted human T cells containing CD28 and CD137 domains. Proc Natl AcadSci USA. 2009;106(9):33603365 .

13- Guoqing W, Lijuan D, Jiasheng W, et al. Advances of CD19-directed chimeric antigen receptor-modified T cells in refractory/relapsed acute lymphoblastic leukemia/experimental hematology \& oncology2017; 6: 10.

14- Shulei YIN, Yizhi YU, Xuetao CAO. Current situation and development trend of CAR-NK anti-tumor research. Chin J Cancer Biother. 2016;23(1): 1-10.

15- June CH, Blazar BR, Riley JL. Engineering lymphocyte subsets: tools, trials and tribulations. Nat Rev Immunol. 2009; 9(10):704-716.

16- Turtle CJ, Hanafi LA, Berger C, et al. CD19 CAR-T cells of defined CD4+: CD8+ composition in adult B cell ALL patients. J Clinlnvestig. 2016; 126(6):2123-2138.

17- Maude SL, Teachey DT, Porter DL, et al. CD19-targeted chimeric antigen receptor T-cell therapy for acute lymphoblastic leukemia.Blood 2015; 125(26): 4017-4023.

18- Scheuermann RH, Racila E. CD19 antigen in leukemia and lymphoma diagnosis and immunotherapy. Leuk Lymphoma. 1995;18(5-6):385-397.

19- Kowolik CM, Topp MS, Gonzalez S, et al. CD28 costimulation provided through a CD19-specific chimeric antigen receptor enhances in vivo persistence and antitumor efficacy of adoptively transferred T cells. Cancer Res. 2006;66(22):10995-11004.

20- Haso W, Lee DW, Shah NN, et al. Anti-CD22-chimeric antigen receptors targeting B-cell precursor acute lymphoblastic leukemia. Blood. 2013;121(7):1165-1174.

21- Scholler J, Brady TL, Binder-Scholl G, et al. Decade-long safety and function of retroviral-modified chimeric antigen receptor T cells. Sci Transl Med. 2012;4(132):132ra153.

22- Grupp SA, Kalos M, Barrett D, et al. Chimeric antigen receptor-modified T cells for acute lymphoid leukemia. N Eng J Med. 2013; 368(16):1509-1518. 
23- Lee DW III, Shah N, Stetler-Stevenson M, et al. Autologouscollected anti-cd19 chimeric antigen receptor (CAR) T cells for acute lymphocytic leukemia (ALL) and Non-Hodgkin's lymphoma (NHL) in children who have previously undergone allogeneic hematopoietic stem cell transplantation (HSCT). Cancer Res. $2013 ; 73$.

24- Hu Y, Wu Z, Luo Y, et al. Potent anti-leukemia activities of chimeric antigen receptor modified T cells against CD19 in Chinese patients with relapsed/refractory acute lymphocytic leukemia. Clin Cancer Res. 2016.

25- Lee DW, Kochenderfer JN, Stetler-Stevenson M, et al. T cells expressing CD19 chimeric antigen receptors for acute lymphoblastic leukaemia in children and young adults: a phase 1 dose-escalation trial. Lancet. 2015;385(9967):517-528.

26- Xu XJ, Tang YM, Song H, et al. Diagnostic accuracy of a specific cytokine pattern in hemophagocytic lymphohistiocytosis in children. The Journal of pediatrics, 2012; 160(6): 984-990.

27- Maude SL, Frey N, Shaw PA, et al. Chimeric antigen receptor T cells for sustained remissions in leukemia. N Engl J Med. 2014; 371(16):1507-1517.

28- Tang YM, Xu XJ. Advances in hemophagocyticlymphohistiocytosis: pathogenesis, early diagnosis/differential diagnosis, and treatment. Scientific World Journal. 2011;11:697-708.

29- Davila ML, Riviere I, Wang X, et al. Efficacy and toxicity management of 19-28z CAR T cell therapy in B cell acute lymphoblastic leukemia. SciTransI Med. 2014;6(224):224:235.

30- Lee DW, Gardner R, Porter DL, et al. Current concepts in the diagnosis and management of cytokine release syndrome. Blood. 2014; 124(2):188-195.

31- Shah NN, Stetlerstevenson M, Yuan CM, et al. Minimal residual disease negative complete remissions following anti-CD22 chimeric antigen receptor (CAR) in children and young adults with relapsed/refractory acute lymphoblastic leukemia (ALL). Blood.2016; 128:650.

32- Drent $E$, Themeli $M$, Renée $P$, et al. Reducing on-target off-tumor effects of CD38-chimeric antigen receptors by affinity optimization. Blood.2016; 128:217.

33- Avanzi MP, Leeuwen D, Li X, et al. IL-18 secreting CAR T cells enhance cell persistence, induce prolonged B cell aplasia and eradicate CD19+ tumor cells without need for prior conditioning. Blood.2016; 128:816.

34- De Oliveira SN, Ryan C, Giannoni F, et al. Modification of hematopoietic stem/progenitor cells with CD19specific chimeric antigen receptors as a novel approach for cancer immunotherapy. Hum Gene Ther. 2013;24(10):824-839.

35- Otáhal P, Průková D, Král V, et al. Lenalidomide enhances antitumor functions of chimeric antigen receptor modified T cells. Oncoimmunology. 2016; 5(4):e1115940. 